\title{
Expression and promoter methylation of Wnt inhibitory factor-1 in the development of oral submucous fibrosis
}

\author{
SHANGHUI ZHOU ${ }^{1}$, LING CHEN ${ }^{2}$, MUBARAK MASHRAH ${ }^{1}$, YUN ZHU $^{1}$, ZHIJING HE $^{3}$, \\ YUHUA HU $^{4}$, TINGXIU XIANG ${ }^{2}$, ZHIGANG YAO ${ }^{5}$, FENG GUO ${ }^{6}$ and CHENPING ZHANG ${ }^{1}$
}

\begin{abstract}
${ }^{1}$ Department of Oral and Maxillofacial-Head and Neck Oncology, Shanghai Ninth People's Hospital, Shanghai Jiaotong University School of Medicine, Shanghai 200011; ${ }^{2}$ Molecular Oncology and Epigenetics Laboratory, The First Affiliated Hospital of Chongqing Medical University, Chongqing 400016; ${ }^{3}$ Department of Oral and Maxillofacial Surgery, The Second Xiangya Hospital, Central South University, Changsha, Hunan 410008; ${ }^{4}$ Department of Oral Pathology, Shanghai Ninth People's Hospital, Shanghai Jiaotong University School of Medicine, Shanghai 20001; ${ }^{5}$ Department of Oral Pathology, Xiangya Stomatological Hospital, Central South University, Changsha, Hunan 410078; ${ }^{6}$ Department of Oral and Maxillofacial Surgery, Xiangya Hospital, Central South University, Changsha, Hunan 410008, P.R. China
\end{abstract}

Received May 29, 2015; Accepted July 6, 2015

DOI: $10.3892 / o r .2015 .4264$

\begin{abstract}
Oral squamous cell carcinoma (OSCC) is a type of head and neck malignancy with a high mortality rate. Oral submucous fibrosis (OSF) is the pre-cancerous lesion of OSCC, whose molecular mechanisms in OSCC tumorigenesis remain largely unclear. Activation of the $\mathrm{Wnt} / \beta$-catenin signaling pathway plays an important role in oral mucous carcinogenesis, although rare mutations of Wnt signaling molecules are found in OSCC, suggesting an epigenetic mechanism mediating aberrant $\mathrm{Wnt} / \beta$-catenin signaling in OSCC. Wnt inhibitory factor-1 (WIF1) is an Wnt antagonist, and its downregulation and methylation have been reported in a number of malignancies. However, the expression and methylation of WIF1 in the development of OSF have yet to be reported. In the present study, we investigated the WIF1 expression level by immunohistochemical staining and semi-quantitative RT-PCR in normal oral, OSF and OSCC tissues, as well as the methylation status by methylation-specific PCR and bisulfite genomic sequencing. The results showed that WIF1 was readily expressed in normal oral mucous tissues, but decreased gradually in OSF early, moderately advanced and advanced tissues, and was less expressed in OSCC tissues. Moreover, WIF1 was able to translocate from the nuclear to cytoplasm in OSF and OSCC tissues. Furthermore, WIFI was frequently methylated in OSCC cases with betel quid chewing habit, but not in normal
\end{abstract}

Correspondence to: Dr Shanghui Zhou or Professor Chenping Zhang, Department of Oral and Maxillofacial-Head and Neck Oncology, Shanghai Ninth People's Hospital, Shanghai Jiaotong University School of Medicine, 639 Zhizaoju Road, Huangpu, Shanghai 200011, P.R. China

E-mail: zhoushanghui@shsmu.edu.cn

E-mail: zhang.chenping@hotmail.com

Key words: WIF1, methylation, tumor suppressor gene, OSF, OSCC oral mucous and different stages of OSF tissues, suggesting WIF1 methylation is tumor-specific in the development of OSF. Thus, the results demonstrated that WIFl is frequently downregulated or silenced by promoter methylation in the carcinogenesis of OSF, which serves as a potential epigenetic biomarker for the early detection of OSCC.

\section{Introduction}

Oral squamous cell carcinoma (OSCC) is a common type of cancer of the head and neck region, with a high mortality rate and rising incidence $(1,2)$. Oral carcinogenesis is a complex multi-step process, undergoing sequential histopathological stages including hyperplasia, oral epithelial dysplasia and final malignant phenotypes $(3,4)$. Despite significant advances in the diagnosis and treatment of OSCC in recent years, 5-year survival rate remains low due to late-stage diagnosis.

Oral submucous fibrosis (OSF), as a pre-cancerous condition (5), is a chronic and potentially malignant disorder, first described in the early 1950s by Pindborg and Sirsat (6). OSF is predominantly prevalent in Indian, mainland China (such as Hunan and Hainan), Taiwan, Bangladesh and Pakistan (7). It is characterized by submucosal fibrosis initially occurring in the oral cavity and then progressively infringing the pharynx and upper esophagus (8). The malignant transformation rate of OSF has been reported in the range of 7-30\% based on the different population (9). The etiology of OSF is multifactorial and remains vague. Chewing of betel quid containing areca nut is the strongest risk factor of OSF, and other factors include tobacco use, alcohol drinking, as well as genetic and immunologic predisposition (10-12). Thus, elucidating the molecular events of OSF malignant progression may be useful in identifying potential biomarkers for the early diagnosis and prevention of OSCC.

Epigenetic modifications including the promoter $\mathrm{CpG}$ methylation and histone modification have been recognized as being crucial events in cancer development, such as genetic 
alterations $(13,14)$. Aberrant promoter methylation leads to the transcriptional silencing of tumor-suppressor genes (TSGs), involved in cell malignant transformation and tumorigenesis. Detection of promoter CpG methylation in human DNA isolated from primary tumor tissues and bodily fluids has become a promising approach for non-invasive screening and early diagnosis of cancer (15). Several TSGs have been identified methylated in OSCC (16-19), such as p16, pl4arf, E-cadherin, O-6-methylgua-nine-DNA methyltransferase (MGMT), deathassociated protein kinase $(D A P K)$, runt-related transcription factor $3(R U N X 3)$, as well as certain Wnt antagonists.

The Wnt/ $\beta$-catenin signaling pathway plays an important role in human malignancies including OSCC $(20,21)$. Wnt inhibitory factor-1 (WIF1) as a secreted WNT antagonist inhibits Wnt/ $\beta$-catenin signaling by directly binding to Wnt proteins (22). However, few studies have focused on the epigenetic disruption of WIF1 in OSF progression. In the present study, we investigated the expression level and methylation status of WIF1, a key antagonist in WNT signaling, in the carcinogenesis of OSF.

\section{Materials and methods}

Tissue specimens. OSCC $(\mathrm{n}=55)$, OSF $(\mathrm{n}=45)$ and normal oral mucosa $(n=15)$ tissue specimens were obtained at the time of surgical resection at the Xiangya Second Hospital and Xiangya Hospital, Central South University (Changsha, China) and Shanghai Ninth People's Hospital, Shanghai Jiaotong University School of Medicine (Shanghai, China) from Juanuary 2013 to June 2014. Informed consent from the patients was obtained under a protocol reviewed and approved by the Institutional Review Boards of the Xiangya School of Medicine or Shanghai Jiaotong University School of Medicine. The clinical diagnosis and pathological stage of OSF was determined in terms of the Pindborg criteria (23). OSCC was diagnosed according to the World Health Organization criteria of 2005. Fifteen normal specimens were obtained from healthy oral mucosa. Forty-five cases of OSF were incident, newly diagnosed without OSCC or neoplastic disease. OSF was classified into 3 grades: early stage $(\mathrm{E}, \mathrm{n}=15)$, moderately advanced stage $(\mathrm{M}, \mathrm{n}=15)$ and advanced stage $(A, n=15)$. The collected tissues were divided into two groups. Tissues in the first group were frozen immediately after careful removal of the tumor mass, OSF tissue in epithelium layer, and grossly normal-looking epithelium, all at $-80^{\circ} \mathrm{C}$. Tissues in the second group were fixed in $4 \%$ buffered formalin solution for pathological diagnosis and immunohistochemical staining. Clinicopathological staging of OSCC was determined by the TNM classification of the International Union Against Cancer in 2009.

Immunohistochemistry. Immunohistochemical staining was performed on $4-\mu \mathrm{m}$ serial sections from formalin-fixed paraffin-embedded specimens. Following deparaffinization and hydration, the slides were treated with endogenous peroxidase in $3 \% \mathrm{H}_{2} \mathrm{O}_{2}$ for $20 \mathrm{~min}$, after which the sections were blocked for $30 \mathrm{~min}$ at $37^{\circ} \mathrm{C}$ with $1.5 \%$ blocking serum in phosphate-buffered saline (PBS) prior to reacting with WIF1 antibody (1:100 dilution, AP2723a; Abgent, San Diego, CA, USA) at $4^{\circ} \mathrm{C}$ in a moist chamber overnight. Negative control slides were duplicate sections in the absence of primary antibodies. To evaluate WIF1 expression, a scoring method was used. A mean percentage of positive tumor cells was determined by the examination of 500 cells in at least 5 areas at $x 400$ magnification. The cells were assigned to 1 of the 5 following categories according to percentage of positive cells (PP): 0) <5\%, 1) 5-24\%, 2) $25-49 \%$, 3) $50-75 \%$, and 4) $>75 \%$. The intensity of the WIF1 staining (SI) then was scored as follows: 0) no, -; 1) weak, +; 2) moderate, ++; and 3) intense, +++ . The final immunoreactive score $($ IRS $=\mathrm{SI}+\mathrm{PP})$ was as follows: -) 0,1 ; +) 2,3 ; ++) 4, 5; and +++$) 6$, 7. The stained tissues were scored blindly with regard to clinical patient data. Statistical analyses were performed with the SPSS 17.0 software. Statistical significance was evaluated by the Chi-square test $\left(\chi^{2}\right)$.

Reverse transcription-polymerase chain reaction. Total RNAs were extracted using TRIzol reagent (Invitrogen Life Technologies, Karlsruhe, Germany) according to the manufacturer's instructions. Reverse transcription polymerase chain reaction (RT-PCR) was performed with the use of a kit from Promega (Madison, WI, USA). Quantitative PCR was performed to detect $W I F 1$ expression, according to the manufacturer's instructions (HT7500 system; Applied Biosystems, Foster City, CA, USA). Primers for the amplification of WIF1 mRNA sequences (accession no. U75285) were synthesized as previously described $(24,25)$. The 257-bp mRNA of WIF1 was amplified by PCR using the primers: forward, 5'-TATGGA TCGATGCTCACCAG and reverse, 5'-CAGAGGGACATT GACGGTTG. GAPDH was used as an internal control. Primers used for GAPDH were: forward, 5'-ATCTCTGCC CCCTCTGCTGA-3' and the reverse, 5'-GATGACCTTGCC CACAGCCT-3'. PCR amplification was performed as follows: denaturation at $94^{\circ} \mathrm{C}$ for $30 \mathrm{sec}$, annealing at $55^{\circ} \mathrm{C}$ for $30 \mathrm{sec}$, and extension at $72^{\circ} \mathrm{C}$ for $30 \mathrm{sec}$ in 32 cycles. The PCR products were visualized on $2 \%$ agarose gels under ultraviolet transillumination.

Methylation-specific PCR and bisulfite genomic sequencing. Bisulfite modification of DNA, methylation-specific PCR (MSP) and bisulfite genomic sequencing (BGS) were performed as previously described (24-27). Bisulfite-treated DNA was amplified with the methylation-specific primer set WIFl-m3: 5'-TGTCGTTTTTATTTTCGTTCGC, WIF1-m4: 5'-CGTTTAAACGACTAAACGCG or the unmethylation specific primer set WIFl-u3: 5'-TTTTTGTTGTTTTTATTT TTGTTTGT, WIFl-u4: 5'-TCCCATTTAAACAACTAAAC ACA. MSP was performed using AmpliTaq Gold (methylation-specific primer: annealing temperature $60^{\circ} \mathrm{C}, 40$ cycles, unmethylation-specific primer: annealing temperature $58^{\circ} \mathrm{C}$, 40 cycles). For BGS, bisulfite-treated DNA was amplified using primers BGS1: 5'-GTTTTAGGGGTTTTTGAGTGTT and BGS2: 5'-CAACTCCCTCAACCAAAACTA. The 463-bp PCR products were cloned into the pCR4-TOPO vector (Invitrogen, Carlsbad, CA, USA), with 8-10 colonies being randomly selected and sequenced.

\section{Results}

Downregulation of WIFl expression at the protein level in the carcinogenesis of OSF. To evaluate WIF1 protein expres- 
Table I. WIF1 expression in the carcinogenesis of oral submucous fibrosis (OSF).

\begin{tabular}{lccccccc}
\hline & & \multicolumn{3}{c}{ WIF1 } & & Mean WIF1 \\
\cline { 3 - 5 } Group & $\mathrm{n}$ & & + & ++ & +++ & WIF1 expression & score \\
\hline Normal & 15 & 1 & 0 & 5 & 9 & $93.3 \%$ & 5.73 \\
OSF & 45 & 12 & 10 & 17 & 6 & $73.3 \%$ & 3.29 \\
E & 15 & 2 & 2 & 8 & 3 & $86.7 \%$ & 4.13 \\
M & 15 & 3 & 3 & 7 & 2 & $80.0 \%$ & 3.60 \\
A & 15 & 7 & 5 & 2 & 1 & $53.3 \%$ & 2.13 \\
OSCC & 55 & 35 & 18 & 2 & 0 & $36.4 \%$ & 1.27 \\
\hline
\end{tabular}

E, early stage of OSF; M, moderately advanced stage of OSF; A, advanced stage of OSF; OSCC, oral squamous cell carcinoma.

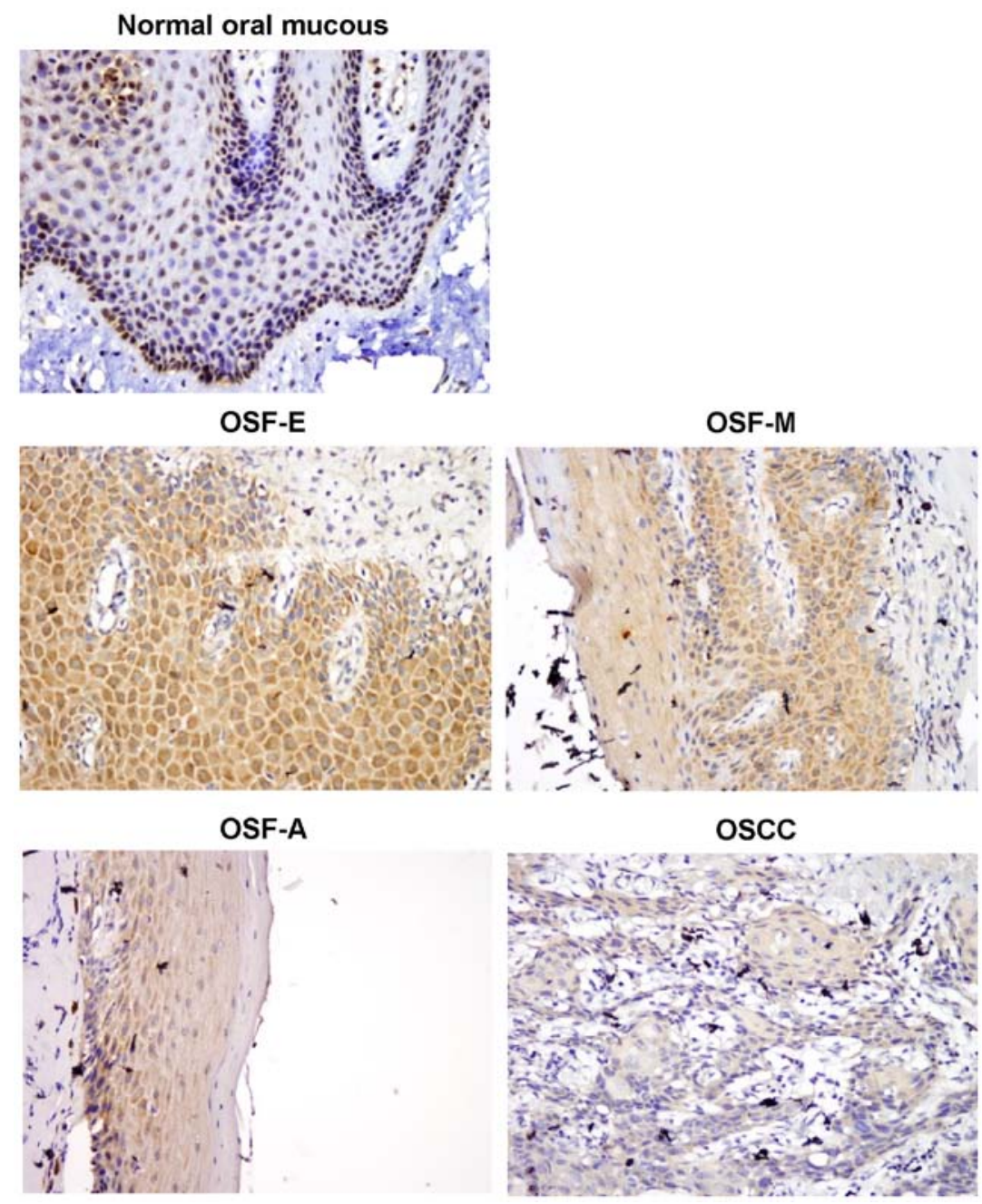

Figure 1. Immunohistochemical staining of WIF1 in normal oral mucosa, OSF and OSCC tissues. Normal oral tissues exhibited strongly positive WIF1 protein expression in the nucleus. OSF early stage and moderately advanced stage tissues showed WIF1-positive expression in the cytoplasm and nucleus. OSF advanced stage tissues showed a weak WIF1 expression in cytoplasmic parts. Primary OSCC showed very weak cytoplasmic immunoreaction for WIF1 (original magnification, $\mathrm{x} 400$ ).

sion, we initially performed immunohistochemical staining using WIF1-specific antibody in normal oral mucous tissues, OSF and OSCC tissues. Fourteen of 15 (93.3\%) normal oral mucous cases showed nuclear WIF1 positivity, while 33 of 45 (73.3\%) OSF tissues showed cytoplasmic WIF1 expression, including tissues from 13 of 15 (86.7\%) early stage, 12 of $15(80 \%)$ moderately advanced stage and 8 of $15(53.3 \%)$ advanced stage, as well as 20 of 55 (36.4\%) OSCC (Fig. 1). The average values of WIF1 expression varied in different tissue samples, including mean score of 5.37 in normal oral mucous tissues, 3.29 in OSF tissues, and 1.27 in OSCC tissues (Table I). WIF1 expression was gradually reduced in normal 

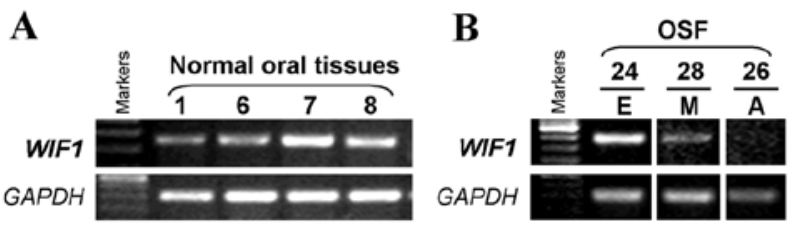

C OSCC and paired adjacent OSF tissues

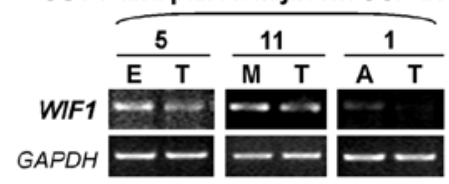

D

OSCC and paired adjacent normal tissues

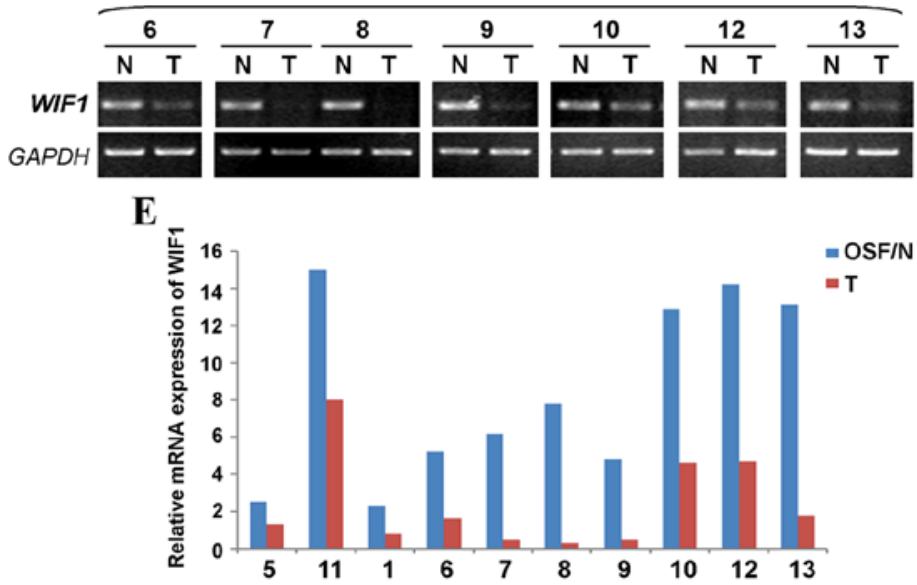

Figure 2. Detection of WIF1 mRNA expression in normal oral mucosa, OSF and OSCC tissues. Semi-quantitative RT-PCR examined WIF1 expression in (A) normal oral mucosa tissues, (B) OSF tissues, (C) OSCC and paired adjacent OSF tissues, as well as (D) OSCC and paired adjacent normal tissues. GAPDH was used as an internal control. (E) RT-qPCR was used to confirm WIF1 expression in representative samples from OSCC and paired adjacent OSF or normal tissues. E, early stage of OSF; M, moderately advanced stage of OSF; A, advanced stage of OSF; N, normal tissue; T, OSCC.

Table II. Correlation between WIF1 expression and clinicopathological characteristics in OSCC cases

\begin{tabular}{|c|c|c|c|c|}
\hline \multirow{2}{*}{$\begin{array}{l}\text { Clinicopathological } \\
\text { characteristics }\end{array}$} & \multirow{2}{*}{$\begin{array}{l}\text { Total } \\
\text { (n) }\end{array}$} & \multicolumn{3}{|c|}{ Results of immunostaining, (n) } \\
\hline & & WIF1 (+) & WIF1 (-) & P-value \\
\hline Age (years) & & & & $>0.05$ \\
\hline$<50$ & 43 & 18 & 25 & \\
\hline$\geq 50$ & 12 & 2 & 10 & \\
\hline Gender & & & & $>0.05$ \\
\hline Male & 52 & 20 & 32 & \\
\hline Female & 3 & 0 & 3 & \\
\hline Tumor site & & & & $>0.05$ \\
\hline Tongue & 40 & 17 & 23 & \\
\hline Others & 15 & 3 & 12 & \\
\hline Primary tumor & & & & $>0.05$ \\
\hline $\mathrm{T} 1+\mathrm{T} 2$ & 12 & 2 & 10 & \\
\hline $\mathrm{T} 3+\mathrm{T} 4$ & 43 & 18 & 25 & \\
\hline \multicolumn{5}{|l|}{ TNM stage } \\
\hline $\mathrm{I}+\mathrm{II}$ & 12 & 7 & 5 & $>0.05$ \\
\hline III+IV & 43 & 13 & 30 & \\
\hline Differentiation grade & & & & $>0.05$ \\
\hline Well & 20 & 5 & 15 & \\
\hline Moderately-poorly & 35 & 15 & 20 & \\
\hline
\end{tabular}

oral tissues, OSF and OSCC tissues $(\mathrm{P}=0.00001)$. However, no statistically significant correlation was observed between WIF1 expression and the clinicopathological characteristics of OSCC ( $>>0.05)$ (Table II). These results suggested that WIF1 is downregulated at a protein level in the carcinogenesis of OSF.

WIF1 mRNA expression is reduced in the carcinogenesis of $O S F$. We also examined WIFl expression at the mRNA level in normal oral mucous tissues, OSF tissues, OSCC and their paired adjacent tissues by semi-quantitative RT-PCR. The presence of the WIF1 mRNA was shown by the 257-bp RT-PCR product. We found that WIF1 was readily expressed in normal oral mucous tissues (Fig. 2A) and OSF early stage tissue, but decreased in OSF moderately advanced stage tissue, while rarely expressed in OSF advanced stage tissue (Fig. 2B). We also detected WIF1 expression in OSCC and their adjacent OSF tissues. The results showed that WIFl was highly expressed in OSF early stage and moderately advanced stage tissues, but markedly downregulated in OSF advanced stage and OSCC tissues (Fig. 2C). We also found that WIF1 was barely detected in OSCC tissues, compared with their paired adjacent normal tissues (Fig. 2D). RT-qPCR also confirmed that a reduced expression of WIF1 mRNA in OSCC tissues, compared to the adjacent normal or OSF tissues (Fig. 2E). Therefore, WIF1 mRNA expression levels are decreased in the carcinogenesis of OSF.

Promoter methylation of WIF1 in the carcinogenesis of OSF. We investigated the possible regulatory mechanism of WIFl reduction in the carcinogenesis of OSF. As promoter methylation mediates transcriptional repression of TSGs, we initially examined the presence of $\mathrm{CpG}$ island (CGI) in the WIFl promoter and exon 1 by bioinformatics analysis. The region spanning the WIF1 promoter and exon 1 fulfilled the criteria of a CpG island (Gardiner-Garden and Frommer, 1987): GC content, 58.8\%; observed/expected $\mathrm{CpG}$ ratio, 0.73; and a total of $46 \mathrm{CpG}$ sites in a 2026-bp region (Fig. 3), thus as a typical CGI.

We detected the promoter methylation of WIF1 in normal oral mucous and OSF tissues, OSCC and their paired adjacent OSF or normal tissues. We found that WIF1 methylation was not detected in 10 normal oral tissues, 10 OSF tissues from early stage, moderately advanced stage and advanced stage (Fig. 4A and B). We also found that WIFl was frequently methylated in 16 of $20(80 \%)$ OSCC tumor tissues, but not any in their paired adjacent normal and OSF tissues (Fig. 4C-E). 


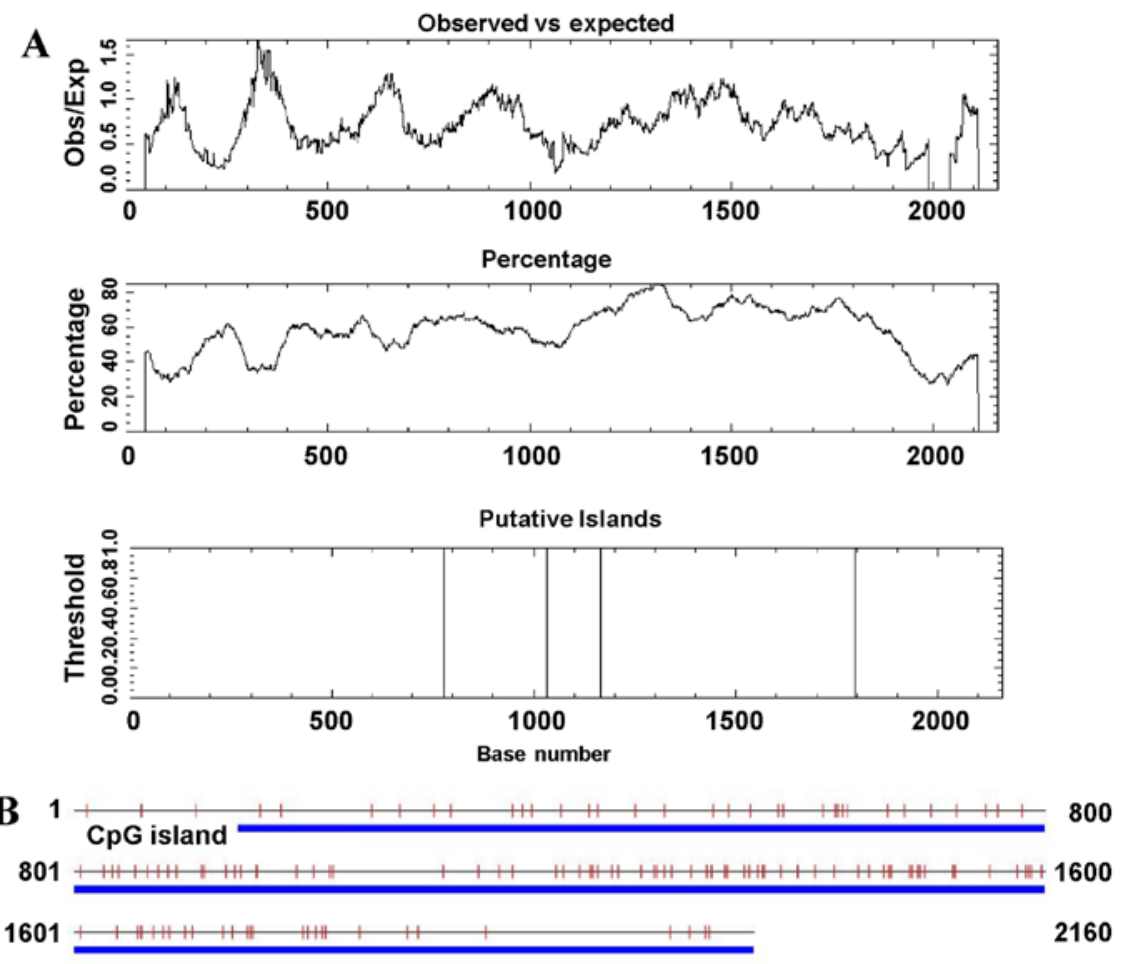

Figure 3. Promoter CpG island analysis of the WIF1 promoter and exon 1 regions by (A) the EMBOSS Cpgplot (http://www.ebi.ac.uk/Tools/seqstats/emboss_ cpgplot/) and (B) CpG island searcher (http://cpgislands.usc.edu/).

A

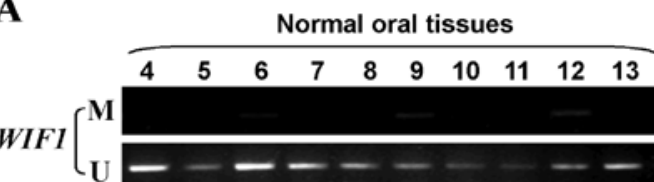

B

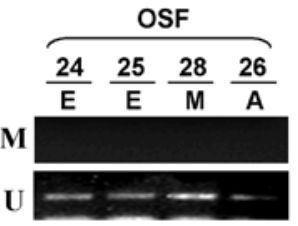

C OSCC and paired adjacent OSF tissues

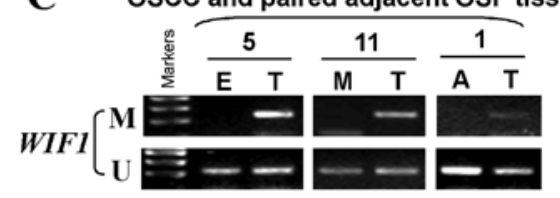

D

OSCC and paired adjacent normal tissues
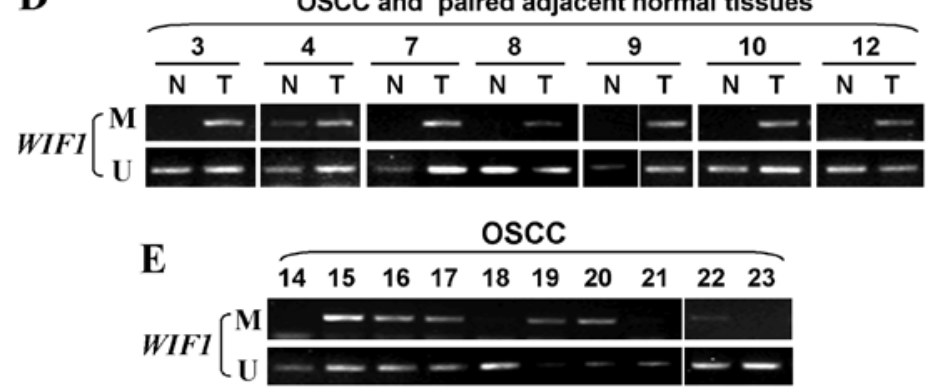

Figure 4. Promoter methylation of WIF1 in normal oral mucosa, OSF and OSCC tissues. MSP was used to detect WIF1 methylation in (A) normal oral mucosa tissues, (B) OSF tissues, (C) OSCC and paired adjacent OSF tissues, (D) OSCC and paired adjacent normal tissues, as well as (E) OSCC tissues. M, methylated; U, unmethylated; E, early stage of OSF; M, moderately advanced stage of OSF; A, advanced stage of OSF; N, normal tissue; T, OSCC.

These results suggested that the promoter methylation of WIF1 is a tumor-specific event in the carcinogenesis of OSF.

Bisulfite genomic sequencing of the WIF1 promoter in OSF and OSCC tumor tissues. To confirm MSP data, high resolution methylation analysis on every CpG site in the WIF1 promoter was carried out by using bisulfite genomic sequencing (BGS). We found that no or very few methylated $\mathrm{CpG}$ sites were detected in representative normal oral mucous tissues, and OSF early stage and moderately advanced stage tissues, while 


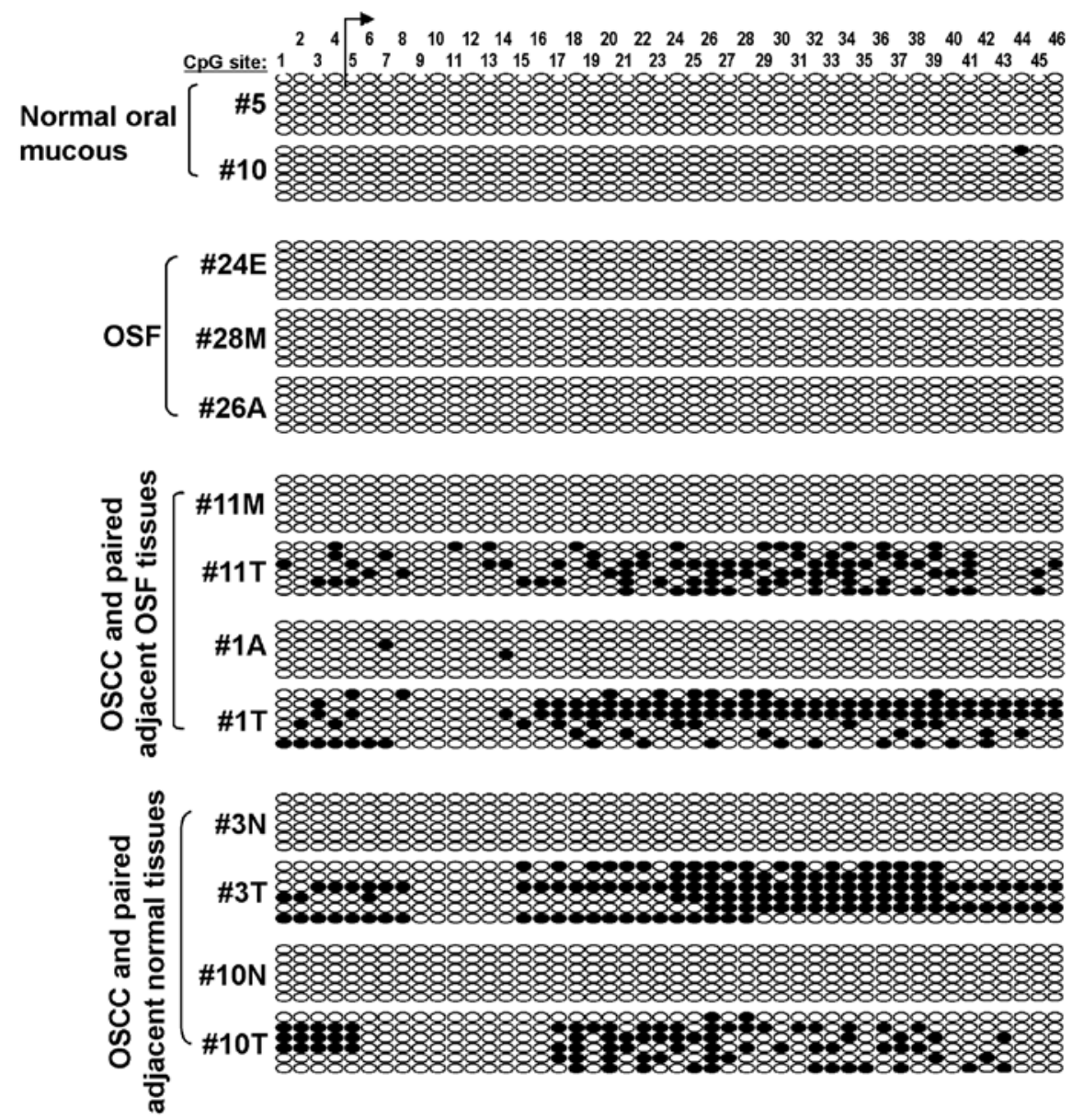

Figure 5. High-resolution mapping of individual CpG sites in the WIF1 CGI by BGS in normal oral mucosa, OSF and OSCC tissues. Circles indicate CpG site. Filled circles indicate methylated sites. Open circles indicate unmethylated sites. Rows indicate individual alleles analyzed.

methylated alleles were detected in OSCC tissues, but not in their paired normal and OSF tissues. The results confirmed the MSP analysis (Fig. 5).

\section{Discussion}

Emerging evidence has shown that the epigenetic silencing of cancer genes via promoter $\mathrm{CpG}$ methylation plays an important role in OSCC pathogenesis. Several genes have been identified to be aberrantly methylated in OSCC, some of which exhibited a potential as biomarkers, for example, E-cadherin promoter methylation is associated with the poor survival in advanced OSCC (16), thus is an ideal epigenetic biomarker for OSCC. Thus, identifying more methylated genes may be useful to develop epigenetic biomarker for the early detection of OSCC.

Wnt signaling pathways include the canonical Wnt, the non-canonical planar cell polarity and the non-canonical Wnt/calcium pathways (28). The canonical Wnt pathway, also known as Wnt/ $\beta$-catenin pathway, accumulates cytoplasmic $\beta$-catenin in and eventual translocates into the nucleus, thus acting as a transcriptional coactivator to activate multiple oncogenic genes, leading to tumorigenesis (29). The $\mathrm{Wnt} / \beta$-catenin pathway is deregulated in various common human cancers (30). Frequent mutations of the signaling molecules in the Wnt/ $\beta$-catenin pathway have been identified in human cancers, including colon, hepatocellular, breast and prostate carcinomas as well as glioblastoma. However, infrequent mutations of APC, Axin1 and $\beta$-catenin genes were reported in OSCC (31), indicating that mechanisms such as epigenetic modulation mediate inactivation of the Wnt/ $\beta$ catenin pathway in OSCC.

WIFl, a secreted Wnt inhibitor, is downregulated and methylated in various carcinomas including hepatocellular (32), nasopharyngeal $(24,33)$, esophageal (5), prostate (34), breast (25), lung (35) and gastrointestinal (36) malignancies, and promotes tumor development and progression by activating $\beta$-catenin. Notably, WIF1 silencing may be an early event in tumorigenesis. Although OSF is the precancerous lesion of OSCC, its molecular mechanisms remain to be elucidated. In the present study, we found that WIF1 either at the protein or mRNA level is highly expressed in normal oral mucous tissues, and is gradually decreased in the different stages of OSF and OSCC tissues. Additionally, WIFI is frequently methylated in OSCC tissues, but not in normal oral mucous tissues, and their paired adjacent normal or OSF tissues. We also detected WIF1 methylation in a panel of OSCC patient tissues with betel quid chewing habit, and observed WIFI methylation. These results are consistent with those of other studies on WIF1 methylation in OSCC in Western populations (18). WIF1 methylation was also reported to be correlated with shorter survival in oral cancer patients (37). Thus, it may become a tumor marker for the early detection of OSCC. Large-scale studies focusing on 
OSF and OSCC samples should be conducted to confirm the potential value of WIFl methylation as an epigenetic biomarker in OSCC in the Chinese population.

In summary, we provide evidence that WIFl is frequently methylated in OSCC in a Chinese population, but not in normal oral mucous and OSF tissues, which is associated with its reduced expression. The present study reveals a novel epigenetic event in the carcinogenesis of OSF, which shed light on the development of a valuable epigenetic biomarker that may be useful for the early detection of OSCC.

\section{Acknowledgements}

The present study was supported by the National Natural Science Foundation of China (no. 81202133) and the Fifth Outstanding Youth Fund of Shanghai Ninth People's Hospital, Shanghai Jiaotong University School of Medicine.

\section{References}

1. Torre LA, Bray F, Siegel RL, Ferlay J, Lortet-Tieulent J and Jemal A: Global cancer statistics, 2012. CA Cancer J Clin 65: 87-108, 2015

2. Braakhuis BJ, Brakenhoff RH and Leemans CR: Head and neck cancer: Molecular carcinogenesis. Ann Oncol 16 (Suppl 2): ii249-ii250, 2005 .

3. Leemans CR, Braakhuis BJ and Brakenhoff RH: The molecular biology of head and neck cancer. Nat Rev Cancer 11: 9-22, 2011.

4. Choi S and Myers JN: Molecular pathogenesis of oral squamous cell carcinoma: Implications for therapy. J Dent Res 87: 14-32, 2008.

5. Pindborg JJ, Murti PR, Bhonsle RB, Gupta PC, Daftary DK and Mehta FS: Oral submucous fibrosis as a precancerous condition. Scand J Dent Res 92: 224-229, 1984.

6. Pindborg JJ and Sirsat SM: Oral submucous fibrosis. Oral Surg Oral Med Oral Pathol 22: 764-779, 1966.

7. Tilakaratne WM, Klinikowski MF, Saku T, Peters TJ and Warnakulasuriya S: Oral submucous fibrosis: Review on aetiology and pathogenesis. Oral Oncol 42: 561-568, 2006.

8. Wollina U, Verma SB, Ali FM and Patil K: Oral submucous fibrosis: An update. Clin Cosmet Investig Dermatol 8: 193-204, 2015.

9. Murti PR, Bhonsle RB, Pindborg JJ, Daftary DK, Gupta PC and Mehta FS: Malignant transformation rate in oral submucous fibrosis over a 17-year period. Community Dent Oral Epidemiol 13: $340-341,1985$

10. Zhang $X$ and Reichart PA: A review of betel quid chewing, oral cancer and precancer in Mainland China. Oral Oncol 43 424-430, 2007.

11. Pillai R, Balaram P and Reddiar KS: Pathogenesis of oral submucous fibrosis. Relationship to risk factors associated with oral cancer. Cancer 69: 2011-2020, 1992.

12. Jian XC, Liu SF, Shen ZH and Yang YH: Histomorphology of oral submucous fibrosis. Report of 24 cases. Chin Med J (Engl) 101: 505-509, 1988.

13. Jones PA and Baylin SB: The fundamental role of epigenetic events in cancer. Nat Rev Genet 3: 415-428, 2002.

14. Jones PA and Baylin SB: The epigenomics of cancer. Cell 128 : 683-692, 2007.

15. Baylin SB and Jones PA: A decade of exploring the cancer epigenome - biological and translational implications. Nat Rev Cancer 11: 726-734, 2011

16. Supić G, Kozomara R, Branković-Magić M, Jović N and Magić Z: Gene hypermethylation in tumor tissue of advanced oral squamous cell carcinoma patients. Oral Oncol 45: 1051-1057, 2009.

17. Towle R, Truong D, Hogg K, Robinson WP, Poh CF and Garnis C: Global analysis of DNA methylation changes during progression of oral cancer. Oral Oncol 49: 1033-1042, 2013.

18. Pannone G, Bufo P, Santoro A, Franco R, Aquino G, Longo F, BottiG, Serpico R, CafarelliB, Abbruzzese A, etal: WNT pathway in oral cancer: Epigenetic inactivation of WNT-inhibitors. Oncol Rep 24: 1035-1041, 2010
19. Ha PK and Califano JA: Promoter methylation and inactivation of tumour-suppressor genes in oral squamous-cell carcinoma. Lancet Oncol 7: 77-82, 2006.

20. Liu F and Millar SE: Wnt/beta-catenin signaling in oral tissue development and disease. J Dent Res 89: 318-330, 2010.

21. Noguti J, DE Moura CF, Hossaka TA, Franco M, Oshima CT, Dedivitis RA and Ribeiro DA: The role of canonical WNT signaling pathway in oral carcinogenesis: A comprehensive review. Anticancer Res 32: 873-878, 2012.

22. Hsieh JC, Kodjabachian L, Rebbert ML, Rattner A, Smallwood PM, Samos CH, Nusse R, Dawid IB and Nathans J: A new secreted protein that binds to Wnt proteins and inhibits their activities. Nature 398: 431-436, 1999.

23. Gupta PC, Sinor PN, Bhonsle RB, Pawar VS and Mehta HC: Oral submucous fibrosis in India: A new epidemic? Natl Med J India 11: 113-116, 1998.

24. Chan SL, Cui Y, van Hasselt A, Li H, Srivastava G, Jin H, Ng KM, Wang Y, Lee KY, Tsao GS, et al: The tumor suppressor Wnt inhibitory factor 1 is frequently methylated in nasopharyngeal and esophageal carcinomas. Lab Invest 87: 644-650, 2007.

25. Ai L, Tao Q, Zhong S, Fields CR, Kim WJ, Lee MW, Cui Y, Brown KD and Robertson KD: Inactivation of Wnt inhibitory factor-1 (WIF1) expression by epigenetic silencing is a common event in breast cancer. Carcinogenesis 27: 1341-1348, 2006.

26. Qiu GH, Tan LK, Loh KS, Lim CY, Srivastava G, Tsai ST, Tsao SW and Tao Q: The candidate tumor suppressor gene BLU, located at the commonly deleted region 3p21.3, is an E2F-regulated, stressresponsive gene and inactivated by both epigenetic and genetic mechanisms in nasopharyngeal carcinoma. Oncogene 23: 4793-4806, 2004.

27. Ying J, Li H, Seng TJ, Langford C, Srivastava G, Tsao SW, Putti T, Murray P, Chan AT and Tao Q: Functional epigenetics identifies a protocadherin PCDH10 as a candidate tumor suppressor for nasopharyngeal, esophageal and multiple other carcinomas with frequent methylation. Oncogene 25: 1070-1080, 2006.

28. Anastas JN and Moon RT: WNT signalling pathways as therapeutic targets in cancer. Nat Rev Cancer 13: 11-26, 2013.

29. Clevers $\mathrm{H}$ : Wnt/beta-catenin signaling in development and disease. Cell 127: 469-480, 2006.

30. Polakis P: The many ways of Wnt in cancer. Curr Opin Genet Dev 17: 45-51, 2007

31. Iwai S, Katagiri W, Kong C, Amekawa S, Nakazawa M and Yura Y: Mutations of the APC, beta-catenin, and axin 1 genes and cytoplasmic accumulation of beta-catenin in oral squamous cell carcinoma. J Cancer Res Clin Oncol 131: 773-782, 2005.

32. Ding Z, Qian YB, Zhu LX and Xiong QR: Promoter methylation and mRNA expression of DKK-3 and WIF-1 in hepatocellular carcinoma. World J Gastroenterol 15: 2595-2601, 2009.

33. Lin YC, You L, Xu Z, He B, Mikami I, Thung E, Chou J, Kuchenbecker K, Kim J, Raz D, et al: Wnt signaling activation and WIF-1 silencing in nasopharyngeal cancer cell lines. Biochem Biophys Res Commun 341: 635-640, 2006.

34. Yee DS, Tang Y, Li X, Liu Z, Guo Y, Ghaffar S, McQueen P, Atreya D, Xie J, Simoneau AR, et al: The Wnt inhibitory factor 1 restoration in prostate cancer cells was associated with reduced tumor growth, decreased capacity of cell migration and invasion and a reversal of epithelial to mesenchymal transition. Mol Cancer 9: 162, 2010.

35. Mazieres J, He B, You L, Xu Z, Lee AY, Mikami I, Reguart N, Rosell R, McCormick F and Jablons DM: Wnt inhibitory factor-1 is silenced by promoter hypermethylation in human lung cancer. Cancer Res 64: 4717-4720, 2004

36. Taniguchi $\mathrm{H}$, Yamamoto $\mathrm{H}$, Hirata $\mathrm{T}$, Miyamoto $\mathrm{N}$, Oki $\mathrm{M}$, Nosho K, Adachi Y, Endo T, Imai K and Shinomura Y: Frequent epigenetic inactivation of Wnt inhibitory factor-1 in human gastrointestinal cancers. Oncogene 24: 7946-7952, 2005.

37. Paluszczak J, Sarbak J, Kostrzewska-Poczekaj M, Kiwerska K, Jarmuż-Szymczak M, Grenman R, Mielcarek-Kuchta D and BaerDubowska W: The negative regulators of Wnt pathway-DACH1, DKK1, and WIF1 are methylated in oral and oropharyngeal cancer and WIF1 methylation predicts shorter survival. Tumour Biol 36: 2855-2861, 2015 